\title{
Percepção ambiental dos moradores de Uberlândia sobre a estação ecológica do Panga - uma visão sob a ótica da economia do meio ambiente
}

A Valoração Ambiental é o processo que busca estimar o valor econômico de recursos naturais através da determinação de equivalência de outros recursos disponíveis na economia. O objetivo desse estudo foi identificar como as pessoas que moram próximas à Estação Ecológica do Panga, situada no município de Uberlândia, Minas Gerais, atribuem valor à esse bem ambiental, através da identificação da DAP e DAR, utilizando-se do Método de Valoração Contingente. Ocorreram aplicação de 300 questionários para o grupo de moradores, no período de 21 de setembro 2015 a 06 de novembro de 2015. Desses, 59\% eram mulheres, 65\% casados, com renda média de R\$ 724,96 mensais e tempo de residência médio de 9,46 anos no local da entrevista. Os moradores apresentaram DAP positiva e DAR negativa; o valor da DAP encontrado foi de R\$ 4.637.520e o da DAR foi de 10.197.360. Após tal análise, foi realizada uma estimação do modelo logit para identificar as variáveis que influenciaram a DAP e DAR dos moradores. Verificou-se que idade, tempo de residência e renda influenciam a DAP positivamente. Já para a DAR, notou-se que os homens têm mais disposição a receber, e pessoas com renda maior possuem menos disposição a receber.

Palavras-chave: Estação Ecológica; Valoração Ambiental; Valoração Contingente; DAP; DAR.

\section{Environmental perception of the Uberlandia habitants about the ecological station of Panga - a view under the environmental economics point}

\begin{abstract}
Environmental valuation is the process that seeks to estimate the economical value of natural resources by determining the equivalence to other resources available in Economy. Therefore, this paper objective is to identify the WTP and PR of two sample groups: area residents and researchers. It were applied 300 questionnaires to the residents group and 11 to the researchers group. The results showed that both groups presented positive WTP and negative PR, the WTP value was R\$ 4.926.960 annual of the residents and $R \$ 5.498 .182$ annual of researchers. It was made logit model estimation to identify the variables that change the WTP and PR of the area residents. The conclusion was: the age, time of residing and incomes changed the WTP values positively. However, referring to PR values, it was notorious that men are more willing to receive, but people with big salaries are not that inclined to receive.
\end{abstract}

Keywords: Ecological Station; Environmental Valuation; Contingent Valuation; WTP; PR.

Topic: Valoração e Economia Ambiental

Reviewed anonymously in the process of blind peer.

William Ferreira Alves

Universidade Federal de Uberlândia, Brasil http://lattes.cnpq.br/1260849374117672 wyl.128@gmail.com

Aracy Alves Araújo

Universidade Federal de Viçosa, Brasil

http://lattes.cnpq.br/5970543576086184

aracy@fagen.ufu.br

Claudionor Ribeiro da Silva

Universidade Federal do Paraná, Brasil

http://lattes.cnpq.br/4808617954565343

crs@ig.ufu.br
Received: 21/07/2017

Approved: 22/10/2017
Referencing this:

ALVES, W. F.; ARAÚJO, A. A.; SILVA, C. R.. Percepção ambiental dos moradores de Uberlândia sobre a estação ecológica do Panga - uma visão sob a ótica da economia do meio ambiente. Revista IberoAmericana de Ciências Ambientais, v.8, n.4, p.309-326, 2017. DOI: http://doi.org/10.6008/SPC2179-6858.2017.004.0025 


\section{INTRODUÇÃO}

A história das unidades de conservação modernas começou com a criação do primeiro parque, o Parque Nacional de Yellowstone, que foi instituído em 1872 com o objetivo de preservar as paisagens "virgens" para as gerações futuras (MCCORMICK, 1992). Em 1885, o Canadá criou seu primeiro parque nacional; a Nova Zelândia o fez em 1894; África do Sul e Austrália em 1898. A América Latina foi um dos primeiros continentes a copiar o modelo de parque nacional sem população humana residente em seus limites; o México criou suas primeiras áreas protegida em 1894; a Argentina, em 1903; o Chile em 1926 (BENSUSAN, 2014). No Brasil, as pressões ambientais levaram a realização da Conferência Brasileira de Proteção à Natureza, em 1934, que teve como um de seus objetivos pressionar o governo federal para a criação de um sistema nacional de unidades de conservação (PÁDUA, 2003).

Porém, apenas em 1988, a Constituição Federal assegurou a todos os brasileiros no seu art. 225, um "meio ambiente ecologicamente equilibrado" e impôs ao poder público o dever de defendê-lo e preservá-lo, além da obrigação de criar áreas protegidas e garantir que elas contribuam para a existência de um "meio ambiente ecologicamente equilibrado" (BENSUSAN, 2014)

A partir dessa base constitucional, o país começava a conceber um Sistema Nacional de Unidades de Conservação (SNUC). O SNUC classifica as unidades de conservação em dois grandes grupos. As Unidades de Proteção Integral e as Unidades de Uso Sustentável. Seguindo a classificação do SNUC, o Brasil, apresenta 10,52\% da superfície do país convertida em Unidades de Conservação, o que representa 101.474.971ha. Do percentual total, 6,34\% são áreas de proteção integral e 3,53\% de uso sustentável, conforme dados compilados pelo Instituto Socioambiental em 2004 (DOUROJEANNI et al., 2007).

As Estações Ecológicas são uma das categorias que compõem as áreas destinadas pelo SNUC a proteção integral. Por lei, as Estações Ecológicas têm como objetivo a preservação da natureza e a realização de pesquisas científicas. Nessas unidades, é proibida a visitação pública, exceto quando o objetivo é educacional e científico. Tais unidades de conservação também foram implantadas no Cerrado brasileiro, o que é justificado pela problemática da conversão de $70 \%$ a $80 \%$ de tal bioma para uso agropecuário (KLINKE et al., 2005). Neste bioma foram estabelecidas as Estações Ecológicas de Uruçuí-Una em Bom Jesus no Piauí, Iquê, em Juína no Mato Grosso, Serra das Araras, em Porto Estrela também no Mato Grosso, Pirapitinga, em Três Marias em Minas Gerais e Serra Geral do Tocantins em Rio da Conceição em Tocantins (ICMBIO, 2016).

Além dessas Estações, em Minas Gerais também encontra-se a Estação Ecológica do Panga (EEP), localizada na bacia do Ribeirão do Panda a $30 \mathrm{~km}$ ao sul do centro urbano de Uberlândia nas coordenadas $19^{\circ}$ $11^{\prime} 40^{\prime \prime}$ S e 48 $19^{\prime} 06^{\prime \prime}$. Possui uma área de 409,5ha e teve sua criação em 1985 quando a área foi comprada pela Fundação Universitária, com o objetivo de que fossem desenvolvidas pesquisas acadêmicas (LIMA et al., 1992).

Pela peculiaridade das Estações Ecológicas - não é permitida a sua utilização como parque, ou fins econômicos - não é difícil supor que a população em geral, que não faz uso direto dos benefícios das Estações Ecológicas, possam ignorar sua existência ou até mesmo sua importância. Assim, questiona-se qual o 
conhecimento da sociedade sobre a Estação Ecológica do Panga e qual o valor que a sociedade atribui a Estação Ecológica do Panga?

O objetivo geral do presente artigo é, portanto, identificar qual o valor que a sociedade atribui à Estação Ecológica do Panga. Especificamente pretende-se identificar a disposição a pagar (DAP) e a disposição a receber (DAR) e a percepção ambiental da EEP pela sociedade. Aceita-se por hipótese que a sociedade reconhece a importância que a estação tem para a preservação e manutenção dos serviços ecossistêmicos, atribuindo DAP positiva e DAR negativa para a Estação Ecológica do Panga.

Com esse dilema para resolver, o desenvolvimento de metodologias econômicas que buscam a avaliação econômica do meio ambiente apresenta-se como uma possível solução. Por mais que essas metodologias possam parecer a mercantilização da natureza, estes estudos buscam estimar o preço de determinados bens e serviços no meio ambiente, servindo como instrumentos para planejamento de Políticas Públicas que busquem melhorias ambientais (MOTTA, 1997). Dessa forma, a Valoração Ambiental é desenvolvida por cientistas econômicos, buscando deter a degradação dos recursos naturais, servindo como mecanismo que estabeleça limites para o desenvolvimento desenfreado e irreversível (MATTOS et al., 2004).

Para um melhor entendimento, este artigo está divido da seguinte forma: a introdução, corrente, que apresenta os objetivos, hipóteses e indicação metodológica; o capítulo seguinte, no qual é discorrido sobre a aplicação da técnica de valoração ambiental em Unidades de Conservação, que é nosso objeto de estudo; o capítulo posterior, que apresenta os procedimentos metodológicos, incluindo uma breve explanação sobre o método de valoração contingente e uma caracterização da área de estudo. Os resultados são apresentados e discutidos no capítulo subsequente e a conclusão no capítulo subsecutivo. Por fim, têmse as referências no capítulo seguido.

\section{REVISÃO TEÓRICA}

\section{Valoração ambiental: método de valoração contingente}

O conceito de valoração ambiental desenvolveu-se dentro da economia neoclássica, tendo para Motta (1997) importância por: "determinar o valor econômico de um recurso ambiental e estimar o valor monetário deste em relação aos outros bens e serviços disponíveis na economia". Para Ortiz (2003), "a valoração ambiental busca avaliar o valor econômico de um recurso ambiental através de determinação do que é equivalente, em termos de outros recursos disponíveis na economia, que estaríamos dispostos a abrir mão de maneira a obter uma melhoria de qualidade ou quantidade do recurso ambiental”.

Mattos et al. (2004) considera a economia ambiental importante para deter a degradação ambiental antes que a situação seja irreversível. Motta (1997) afirma que ao atribuir valor econômico, será possível mostrar condições socioeconômicas que os critérios ecológicos e ambientais isolados não conseguiriam responder. $\mathrm{O}$ valor econômico dos recursos naturais pode ser separado em dois grandes grupos: os bens e serviços ambientais que apresentam o seu valor por estarem relacionados ao seu consumo, gerando o seu valor de uso (VU); e o valor de não-uso (VNU) que representa o valor de existência (VE) que advém de uma 
posição moral, cultural, ética e altruísta em relação ao direito de existências de espécies não humanas ou a preservação de riquezas naturais sem representar o uso atual ou futuro para o indivíduo (MOTTA, 1997).

O Valor de Não-Uso (VNU) nos leva a criação de um mercado hipotético para captar a disposição a pagar (DAP) ou a receber (DAR) da população pela preservação dos recursos ambientais, utilizando do método de função de demanda, do qual a disponibilidade tem relação com a disposição de pagamento para a preservação de um bem natural (MOTTA, 1997).

Sabendo-se dos valores de uso e não-uso dos recursos ambientais, pode-se proceder à Valoração Econômica, utilizando-se de diferentes métodos, que são aplicados de acordo com o objetivo da valoração e nesse processo busca-se contemplar as melhores formas de análise social de custo-benefício e cada método apresenta sua eficiência específica para determinado caso (MOTTA, 1997).

Os métodos de valoração ambiental são divididos entre métodos diretos e indiretos. Nascimento et al. (2013) conceitua os métodos diretos como os que procuram inferir sobre as preferências dos indivíduos por bens ou serviços ambientais a partir de perguntas feitas diretamente às pessoas. Já os métodos de valoração indiretos são aqueles que inferem sobre o valor econômico do recurso ambiental a partir da observação da preferência revelada do indivíduo em mercados correlacionados ao ativo ambiental (MAY et al., 2003).

Entre os métodos diretos, tem-se os que obtém a DAP diretamente (Método de Valoração Contingente) e os que obtém a DAP indiretamente (Preços Hedônicos e Custo de Viagem). Entre os métodos indiretos de valoração, tem-se o da produtividade marginal, o método dos custos evitados, custos de controle, custos de oportunidade e custos de reposição.

Nesse artigo, nos deteremos na análise e explanação do Método de Valoração Contingente - MVC, que será utilizado nesse trabalho. O método de Valoração Contingente é um método de DAP direta e busca estimar o valor de existências de bens e serviços ambientais de acordo com as preferências individuais, uma vez que as pessoas possuem diferentes graus de preferência ou gosto por diversos bens ou serviços. Dessa forma, busca-se chegar a um valor de existência de determinado produto por meio da criação de cenário hipotético que se aproxime as condições do real (MOTTA, 1997).

Mota (2001) afirma que o MVC tem o seu desenvolvimento com a aplicação de surveys, tendo por finalidade a captação dos desejos, preocupações, percepções, comportamentos e as atitudes das pessoas em relação à preservação de um recurso natural, ou mudança ambiental. Dessa forma, diferencia-se dos outros métodos de valoração por utilizar de pesquisa de campo para estimar os benefícios e custos ambientais, não recorrendo a preços relacionados em mercados recorrentes, como reporta Borger (1995), criando-se um cenário hipotético que se aproxime das condições reais, para a formação de um valor de existência de determinado bens e serviços ambientais (MOTTA, 1997).

A criação dos cenários hipotéticos serve para que ao se aplicarem os questionários e entrevistas com os usuários de determinados recursos ambientais as condições a eles apresentadas se aproxime da realidade. Dessa forma, tem-se a possibilidade de estimar a Disposição a Pagar (DAP) e/ou a Disposição a Receber (DAR) 
das pessoas que frequentam ou de que algumas formas são afetadas pela existência do recurso (OBARA, 1999).

Com a consolidação da base teórica por Hanemann (1984) o MVC foi incluído entre os métodos aceitáveis, sendo também aprovado para medir benefícios de danos sob 'The Comprehensive environmental Response, Compensation and Liability of 1980' (Superfund) - Department of the Interior (1986) (BORGER, 1995). Além disso, dada a sua aceitabilidade, agentes financeiros internacionais, como o BIRD (Banco Mundial), e o BID (Banco Interamericano de Desenvolvimento) têm utilizado a metodologia para a estimativa de benefícios econômicos e estudo de viabilidade de projetos a serem financiados por tais instituições (BORGER, 1995). Sua aplicação tem se tornado cada vez mais constante em processos judiciais para pagamento de indenizações a danos causados ao ambiente, caminhando para constituir-se num parâmetro confiável para os agentes econômicos e jurídicos (MAIA, 2002).

Duas aplicações clássicas do MVC para fins jurídicos foram para os casos do vazamento dos quase 260 mil barris de petróleo do navio Exxon Valdez em Prince Willian Sound, Alasca, em 1989, e a estimação dos benefícios gerados pelo aumento da visibilidade no Grand Canyon, com a redução da névoa causada pela emissão de dióxido sulfúrico, em 1991 (MAIA, 2002). Nos anos posteriores a tais eventos, ocorreu a popularização do MVC, decorrente de seu potencial como possibilidade de captar o valor de diferentes tipos de serviços ecossistêmicos, além da mensuração de valores não associados ao uso atual ou potencial, conhecido como valor de uso passivo (CARSON, 2000, citado por ANDRADE, 2013).

Diante do exposto, percebe-se que os métodos de Valoração Ambiental são importantes para o desenvolvimento de políticas públicas, já que procuram estimar um valor monetário para os bens e serviços ambientais (MOTTA, 1997). Com essa premissa, é importante o estabelecimento do Valor de Existência atribuído pelos agentes econômicos envolvidos na Estação Ecológica do Panga, pois a mesma possui grande representatividade ambiental do Cerrado brasileiro (SCHIAVINI et al., 1989).

\section{Aplicações do MVC: uma revisão de literatura}

Nesse tópico serão apresentados resultados dos principais trabalhos desenvolvidos no Brasil com a aplicação dos conceitos de valoração ambiental para Unidades de Conservação, que são o objeto de nosso estudo. Nesse sentido, Obara (1999) percebeu que com o avanço da problemática ambiental e a necessidade de desenvolvimento de estudos para melhor gestão de unidade de conservação, utilizou em seu trabalho o MVC na Estação Ecológica de Jataí, uma Unidade de Conservação, localizada no município de Luiz Antônio, no Estado se São Paulo.

O objetivo de seu trabalho foi estimar o valor monetário que a população adulta do município de Luiz Antônio estaria disposta a pagar para garantir a proteção e a conservação da Estação Ecológica de Jataí, além de estimar o valor total obtido. Como resultado, Obara (1999) concluiu que a população do município de Luiz Antônio apresentou uma DAP anual de R\$49.034,70 para visitar e conservar a Estação Ecológica e o Valor de uso e o Valor de Não-Uso da Estação Ecológica de Jataí foram estimados em, respectivamente, $\mathrm{R} \$ 9.547,05$ e 
$R \$ 39.487,65$. Os valores de herança, de existência e de opção foram estimados em, respectivamente, $R \$ 16.755,15 ; R \$ 13.798,36$; e $R \$ 8.934,12$.

Silva et al. (2004) buscou, em seu trabalho, determinar o valor econômico total do parque 'Chico Mendes', situado na cidade de Rio Branco, no Acre. Como metodologia, Silva et al. (2004) utilizou o MVC e observou que $32 \%$ dos entrevistados não se dispuseram a contribuir com nenhum valor para manutenção e preservação do parque, consequentemente, tendo uma manifestação de $68 \%$ para a contribuição.

Silva et al. (2004) utilizou o modelo logit para identificar as variáveis que influenciaram a DAP. Observou que a probabilidade média de o indivíduo aceitar contribuir com a manutenção e conservação do parque é de 24,12\%. Em relação à renda familiar, verificou-se que o efeito marginal dessa variável sobre a probabilidade de o indivíduo pagar é pequena, pois uma variação na renda familiar do indivíduo em uma unidade monetária acarreta um impacto de 0,0092 pontos percentuais.

Já Cirino et al. (2008) realizou estudos na Área de Proteção Ambiental (APA) São José, localizada na mesorregião do Campos das Vertentes, no sudoeste do estado de Minas Gerais. Apesar de ser uma área de proteção desde 1981, a mesma vem sofrendo com degradações ambientais. O trabalho utilizou-se de MVC e constataram que $59,8 \%$ dos entrevistados estariam dispostos a contribuir, ao passo que $40,2 \%$ não mostraram esse interesse.

Cirino et al. (2008) concluíram que os fatores que influenciaram mais significativamente a DAP, foram: renda familiar, idade e conhecimento prévio acerca das degradações que o ativo em estudo vem sofrendo nos últimos anos. Cirino et al. (2008) constataram que as sociedades envolvidas no processo valorativo, ao se disporem a pagar uma taxa pública mensal, reivindicam políticas públicas efetivas de melhoria e conservação da APA São José.

Ao valorar a Serra de São José, composta por uma APA e um Refúgio da Vida Silvestre (REVS), ambas localizada no Estado de Minas Gerais, nas cidades históricas de Tiradentes, São João del Rei e Prados, bem como nos municípios de Santa Cruz de Minas e Coronel Xavier Chaves, Vieira (2009) constatou por meio do MVC que, $72,48 \%$ dos moradores estariam dispostos a pagar e $75,23 \%$ estariam dispostos a trabalhar voluntariamente.

Vieira (2009) encontrou suas maiores DAP pertenciam aos entrevistados que recebiam, individualmente, 3,5 salários mínimos por mês, possuíam ensino técnico, estavam na faixa etária 18-25 anos, eram do sexo feminino, visitavam a serra atualmente e pretendiam visitá-la no futuro. Como resultado da DAP, Vieira (2009) estimou o valor econômico em $\mathrm{R} \$ 10,00$ por mês para melhorarem o fluxo dos serviços ambientais da Serra de São José. Já o valor mediano da Disposição ao Trabalho Voluntário (DATV) individual foi de $\mathrm{R} \$ 22,64$ por mês. Ao multiplicar estes valores pelo de habitantes residentes na zona urbana de Tiradentes, Vieira (2009) teve como resultado o valor de $R \$ 588.840,00$ e $R \$ 1.333 .133,76$.

Volanova et al. (2010) desenvolveu trabalho em um parque urbano tendo por objetivo identificar a disposição dos usuários do 'Parque da Cidade Mãe Bonifácia' em Cuiabá/MT, por meio do método de Valoração Contingente. Observou-se que os visitantes com maior DAP possuem 1ㅇg grau completo (62,5\%), 
seguidos pelos que possuem segundo grau incompleto $(60,0 \%)$ e dos que possuem ensino superior incompleto (56,5\%). Entre os que têm o 1 o grau incompleto o percentual de dispostos a pagar é de $28,6 \%$, e entre os que possuem curso superior completo, $28,3 \%$. A menor disposição a pagar foi encontrada entre os que possuem segundo grau completo, com apenas 1,9\%. Volanova et al. (2010) observou também que 72,5\% dos entrevistados não se dispuseram a contribuir com nenhum valor para manutenção e preservação do parque, sendo apenas $27,5 \%$ disposto a contribuir.

Silveira et al. (2013) entende que a valoração econômica dos ativos ambientais é importante critério que subsidia a tomada de decisões na definição de políticas que gerenciam os recursos naturais. Nesse sentido, Silveira et al. (2013) buscou valorar a Área de Preservação Ambiental Estadual da Cachoeira das Andorinhas, importante Unidade de Conservação do Município de Ouro Preto/MG. Para estimar o valor ambiental, Silveira et al. (2013) utilizou do MVC, junto com a técnica conhecida como referendum, para obter os indicadores discretos da DAP. Os resultados indicaram que $34,3 \%$ dos entrevistados estariam dispostos a contribuir financeiramente, ao passo que $57,12 \%$ não contribuiriam.

Ao verificar as variáveis que influenciaram na DAP positiva, os autores encontraram que o efeito marginal da variável renda familiar per capita, cuja média na amostra foi de $\mathrm{R} \$ 607,48$, indicou que o aumento de uma unidade monetária na referida variável provoca elevação de 0,016 ponto percentual sobre a probabilidade de uma resposta afirmativa à valoração contingente.

Em relação à idade, Silveira et al. (2013) observou que se teve um efeito marginal negativo, embora pequeno, indicando que a elevação em um ano de vida do entrevistado reduz a probabilidade de pagamento em 0,54 ponto percentual. Outro fato importante constatado por Silveira et al. (2013) foi que o efeito marginal da variável referente à questão se o indivíduo já tinha ouvido falar na APA, em respostas positivas a essa pergunta aumentam a probabilidade de pagamento em 21,79 pontos percentuais.

Para Silveira et al. (2013), tal resultado revela que o fato de o indivíduo possuir vaga noção sobre a importância do patrimônio natural do ativo em questão tem forte influência sobre as chances de obtenção de uma contribuição positiva. Silveira et al. (2013) destacou como importante o resultado obtido pela variável relacionada à escolaridade dos indivíduos, onde está não se mostrou estatisticamente significativa para o pagamento. Tal fato contraria o esperado, ou seja, o grau de instrução influenciando positivamente a probabilidade de uma resposta positiva à valoração ambiental.

Os trabalhos apresentados mostraram que existem esforços dos pesquisadores em estimar valores para os ativos ambientais presente em unidades de conservação por meio do método de valoração contingente. Ao estimar o valor ambiental, tais trabalhos buscam servir de instrumento para a conscientização e melhoria das condições ambientais em unidades de conservação.

\section{METODOLOGIA}

Para identificar o valor ambiental que o grupo de moradores atribuem para a EEP, utilizou-se do Método de Valoração Contingente (MVC). Assim, foi necessário, como preconiza o método, a elaboração de um questionário e de um cenários hipotético, para que ao se aplicarem os questionários as condições a eles 
apresentadas se aproxime da realidade. Dessa forma, tem-se a possibilidade de estimar a Disposição a Pagar (DAP) e/ou a Disposição a Receber (DAR) das pessoas que frequentam ou de que algumas formas são afetadas pela existência do recurso (OBARA, 1999).

Os questionários elaborados foram divididos em 6 grupos de questões, cada qual com o intuito de obter informações importantes dos entrevistados. O primeiro grupo destinou-se a colher informações quanto ao nome dos entrevistados (não obrigatório). Imediatamente foram questionados quanto ao sexo biológico, idade e Estado Civil fechando o bloco de perguntas. No segundo grupo foram agrupadas questões que tiveram como intuito a obtenção de informações quanto às condições sociais e econômicas das quais os entrevistados estavam inseridos.

Já o terceiro grupo de questões, buscou compreender qual o nível de consciência ambiental dos entrevistados. O quarto grupo buscou estimar o grau de conhecimento com que os indivíduos tinham sobre a EEP. O quinto e o sexto grupo foram responsáveis por obter a DAP e a DAR, respectivamente, dos entrevistados.

Para estimar o valor ambiental da Estação Ambiental do Panga pelo MVC, foram aplicados questionários para os residentes próximos da Estação Ecológica do Panga, uma vez que para Obara (1999) são estes moradores que desenvolvem interação direta ou indireta com o bem a ser valorado, e são deles a principal condição de beneficiados com a manutenção dos bens e serviços fornecidos.

A área amostral está totalmente situada no Município de Uberlândia/MG e como o município é de grande extensão territorial, possuindo grande quantidade de moradores, decidiu-se trabalhar segundo a metodologia de Barbisan et al. (2009), onde se tem o ponto central do objeto a ser valorado e a partir desse ponto traça-se a área compreendida por um raio, sendo esta a localização da população em que são aplicados os questionários.

Dessa forma, para identificar a área que contém os moradores a serem entrevistados, foi feito o levantamento de imagens digitais da área em que se encontra a Estação Ecológica do Panga. O ponto central da área da EEP foi levantado em campo, obtendo as coordenada UTM, no sistema WGS 84, Fuso 22, E = 0781949m e N = 7875435m, coletadas com um GPS Garmin, modelo GPSmap 76CSx.

Com a utilização do software ENVI (Environment for Visualizing Imagens), foi possível inserir as coordenadas UTM de localização do ponto central e o raio de interesse $(30 \mathrm{~km})$, estimando a área amostral sobre uma imagem digital do satélite Landsat 8, coletada no dia 04/10/2014, na faixa 221, ponto 73 e com resolução espacial de 30m, sob o código LC82210732014277LGN00. O produto gerado (área amostral) pode ser observado na figura 1.

Para se chegar à amostra da população de moradores, utilizou-se de equação estatística de amostra aleatória simples. Utilizou-se de Equação 01, para chegar à estimação da população amostral: $n=$ $\frac{N \cdot Z^{2} \cdot p \cdot(1-p)}{Z^{2} \cdot p \cdot(1-p)+e^{2} \cdot(N-1)}$. Na equação, $n$ representa a amostra aleatória a ser calculada; $N$ é a população total considerada com 18 mil habitantes; $Z$ é a variável normal padronizada associada ao nível de confiança de $90 \%$; $p$ é a verdadeira probabilidade do evento, e é o erro amostral (5\%). 


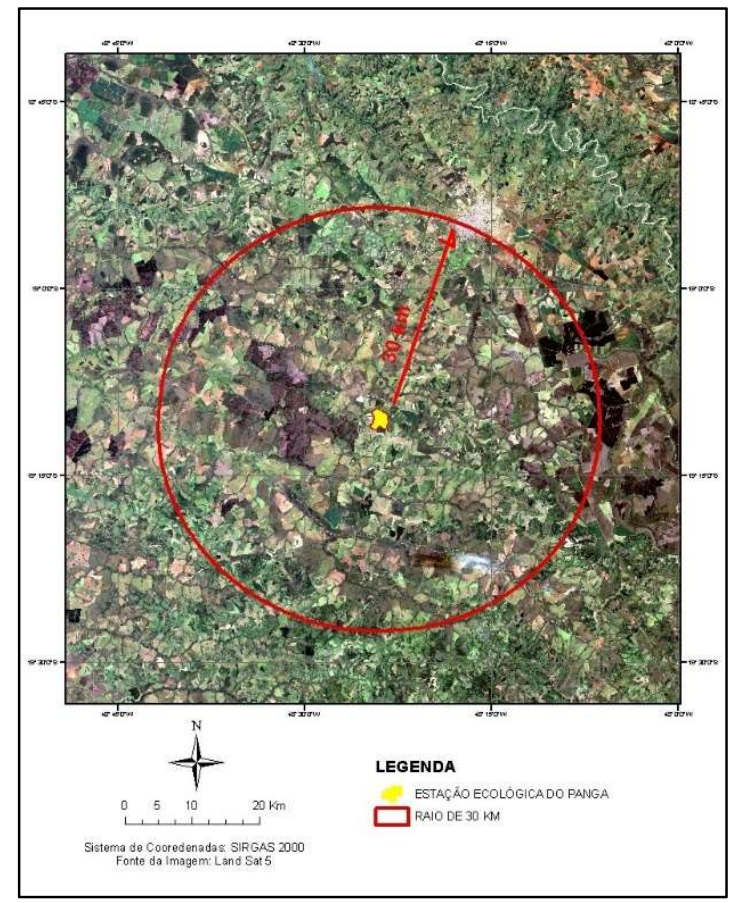

Figura 1: Área de abrangência da população amostral de moradores da EEP, Uberlândia/MG, 2015.

Após o cálculo, chegou-se ao número amostral de 267 questionários a serem aplicados. Mas, buscando uma maior exatidão no nível de confiança, foram aplicados 300 questionários, tendo, dessa forma, um nível de confiança maior que $90 \%$ e inferior a 95\%. Para compreender os efeitos das variáveis dos distintos grupos no seu padrão de DAP e DAR, foi utilizado o método de função de probabilidade logística acumulada (Modelo Logít) (PINDYCK et al., 2004).

Tabela 1: Variáveis utilizadas para estimação do modelo logit e sinais de coeficiente esperados, Uberlândia/MG, 2016.

\begin{tabular}{|c|c|c|}
\hline \multirow{2}{*}{ VARIÁVEL } & \multicolumn{2}{|c|}{ SINAL ESPERADO DO COEFICIENTE } \\
\cline { 2 - 3 } & DAP & DAR \\
\hline Sexo & Negativo & Positivo \\
\hline Idade & Negativo & Positivo \\
\hline dist_eep_km & Negativo & Negativo \\
\hline temp_resid & Positivo & Negativo \\
\hline infra_urbana & Positivo & Positivo \\
\hline num_moradores & Negativo & Negativo \\
\hline Lrendafam & Positivo & Negativo \\
\hline Escola & Positivo & Negativo \\
\hline sustentabilidade & Positivo & Negativo \\
\hline serv_ambi & Positivo & Negativo \\
\hline imp_preserv & Positivo & Negativo \\
\hline Eep & Positivo & Negativo \\
\hline eep_preservação & Positivo & Positivo \\
\hline eep_area_preserv & Positivo & Positivo \\
\hline out_atv_eep & Negativo & \\
\hline exp_eep_emp & Negativo & \\
\hline & & \\
\hline
\end{tabular}

O modelo Logit, é um modelo de probabilidade em que a variável dependente é dicotômica, ou seja, assume valores 0 e 1 . A chance de ocorrência do evento, neste caso, será a chance de um indivíduo contribuir (1) e não contribuir (0). E, dessa forma, será avaliada a disposição a pagar e a receber como uma função das variáveis quantitativas e qualitativas que serão extraídas do questionário. A probabilidade logística acumulada - Modelo Logit - é definida por Fávero et al (2009) conforme as equações: $f(Z)=\frac{1}{1+e^{-(z)}}$, sendo 
Z: $Z=\operatorname{Ln}\left(\frac{P i}{1-P i}\right)=\propto+\beta_{1} X_{1}+\beta_{2} X_{2}+\ldots+B_{k} X_{k}$, em que $P i$ é a probabilidade de ocorrência de um evento i; $X$ representa o vetor das variáveis explicativas e $\alpha$ e $\beta$ são os parâmetros/coeficientes do modelo.

O termo $\operatorname{Ln}\left(\frac{P i}{1-P i}\right)$ é chamado de Logit e o termo $\left(\frac{P i}{1-P i}\right)$ é a chance de ocorrência do evento. Foi utilizado o Software Excel, da Microsoft Corp. para tabulação dos dados e o software STATA (versão 11) da STATA Corp. para estimação do modelo Logit. Na tabela 1, têm-se as variáveis que foram inseridas no modelo para identificação de quais influenciaram a DAR e a DAP dos indivíduos.

Como observado na Tabela 01, espera-se que se tratando de DAP, as variáveis, temp_resid, infra_urbana, Irendafam, escola, sustentabilidade, serv_ambi, eep, eep_preservação e eep_area_preserv apresentem coeficiente positivo. As demais variáveis, espera-se que o coeficiente seja negativo. Quanto a DAR, espera-se que sexo, dist_eep_km, num_moradores, out_atv_eep e exp_eep_emp apresentem coeficiente positivo, as demais espera-se negativo.

\section{RESULTADOS E DISCUSSÃO}

\section{Características da população e percepção ambiental}

Foram entrevistadas 300 pessoas; destes, $59 \%$ eram do sexo feminino e $41 \%$ eram do sexo masculino. A média da idade ficou em 39 anos. A faixa etária compreendida entre os 26 até os 33 anos apresentou o maior percentual de entrevistados, sendo $22 \%$ dos entrevistados. O segundo maior percentual ficou com a faixa etária de $42-50$ anos, totalizando $21 \%$ dos entrevistados. Quanto ao estado civil, observou-se que $65 \%$ dos entrevistados, estavam em situação de matrimônio. Os solteiros foram $22 \%$ dos entrevistados. Separados e viúvos, representavam $7 \%$ e $6 \%$ respectivamente.

Em se tratando do grau de escolaridade, constatou-se que a maioria dos entrevistados possui o segundo grau incompleto, totalizando $28 \%$ dos entrevistados. Entrevistados com Primeiro Grau Incompleto e Segundo Grau Completo representam a segunda e terceira maior incidência de formação escolar dos entrevistados, representando $24 \%$ e $21 \%$, respectivamente. Observou-se um baixo nível de formação superior, pois os respondentes que indicaram possuir Terceiro Grau Completo e o Terceiro Grau Incompleto representam apenas $7 \%$ e 6\%, respectivamente. A renda média individual foi de R\$724,96 por mês. Tal valor é inferior ao salário mínimo referente ao período da aplicação dos questionários, que era de $\mathrm{R} \$ 788,00$.

Também se questionou aos entrevistados quanto tempo residiam no local de moradia. Encontrou-se como média 9,46 anos de residência. Do total de 300 entrevistados, 38\% residiam de 1 a 5 anos. Apenas 5\% dos moradores residiam há mais de 26 anos onde foram entrevistados, mostrando que as ocupações são recentes.

Após a identificação dos entrevistados, os mesmos foram questionados em relação à percepção ambiental. Nesse sentido, Vieira (2009) ressalta que para o bom desenvolvimento do método de valoração contingente, é necessário que os entrevistados entendam que qualquer dano ao bem ambiental influencia em seu bem-estar. Com o intuito de detectar tal compreensão, os entrevistados foram questionados se para 
eles o meio ambiente é importante para a manutenção da vida na Terra e se danos ambientais, como queimadas, poluição de águas e desmatamentos, podiam prejudicar sua qualidade de vida e saúde. Como resultado observou-se que todos (100\%) os entrevistados compreendem a importância do meio-ambiente para a vida na Terra e os danos ambientais são prejudiciais para a qualidade de vida.

Um dos temas correntes da preservação e conservação ambiental é a sustentabilidade, identificouse que $96 \%$ dos entrevistados já tinham ouvido falar desse termo, mostrando que existe minimamente um conhecimento sobre a problemática ambiental. Em relação a noção de serviços ambientais, somente $20 \%$ disseram conhecer o termo.

Para $87 \%$ dos entrevistados a preservação de áreas naturais é muito importante, e $13 \%$ consideram a preservação como importante, o que mostra que a população entrevistada possui entendimento sobre a importância de se preservação as áreas naturais. Provavelmente, um dos principais motivos para este reconhecimento da importância ambiental é decorrente do avanço nas discussões da problemática acerca do meio ambiente.

Em relação ao conhecimento dos entrevistados sobre a EEP, constatou-se que $67 \%$ já tinham ouvido falar da estação, porém, 69\% não sabia da importância da mesma para a preservação da vegetação nativa do Cerrado. Demonstra-se que mesmo sabendo da sua existência, poucos entrevistados sabiam da importância da mesma como importante área de preservação do Cerrado brasileiro. Como a EEP não é um parque aberto, esperou-se que o número de entrevistados que soubessem da existência da estação fosse inferior ao apresentado, mas infere-se que um dos motivos para esse conhecimento seja decorrente da localização dos bairros, localizados próximo à rodovia que leva ao distrito de Miraporanga e a cidade de Campo Florido, passando neste percurso na entrada da EEP.

Após a identificação do nível de conhecimento dos respondentes sobre a EPP e meio ambiente, algumas outras informações foram repassadas, como a seguir: a Estação Ecológica do Panga se situa na Bacia do Ribeirão do Panga, a $30 \mathrm{~km}$ ao sul do centro urbano do município de Uberlândia/MG. Possui uma área de 409,5ha e apresenta grande representatividade dos diversos tipos de vegetação encontrados na região do Cerrado do Brasil Central, como os campestres, savânicas e florestais. Além da presença de aves, mamíferos, répteis, roedores entre outros animais típicos do Cerrado brasileiro.

Informados sobre isto, foram questionados se a EEP se apresentava como importante para eles. Tal questionamento teve como resultado que para $100 \%$ dos entrevistados a EEP apresentava-se importante. Também foram informados que por Lei, dentro da EEP é proibida qualquer tipo de atividade humana, com exceção da pesquisa científica. Logo em seguidas questionados se os mesmos queriam ou não que fossem permitidas outras atividades na EEP.

Dos 300 entrevistados, 59\% gostariam que fossem desenvolvidas outras atividades na EEP, mostrando que existe uma vontade social, de que a área seja utilizada para outros fins, tornando-se um espaço apropriado pela sociedade em geral. Entre as atividades que poderiam vir a serem desenvolvidas na EPP, $48,5 \%$ os respondentes gostariam que fossem desenvolvidas atividades voltadas para a educação ambiental; $26 \%$ defendiam que a EEP fosse transformada em parque aberto e $25,5 \%$ que atividades de 
turismo ecológico fossem desenvolvidas na mesma. Quando questionados sobre a possibilidade de desenvolver atividades dentro da EPP direcionadas para a exploração de recursos naturais, 67\% dos entrevistados consideraram que essa alternativa não é interessante, mesmo que tais atividades trouxessem mais empregos para a região.

\section{Análise da Disposição a Pagar e Disposição a Receber}

Para identificar a DAP dos entrevistados, foi formulado, como preconiza o método de Valoração contingente, a formulação de um cenário hipotético em que se supunha que um avanço da problemática ambiental atingisse a EEP e que, se para controlar tal avanço, o entrevistado estaria disposto a contribuir com uma quantia mensalmente por meio de pagamento por boleto para a Associação de Moradores Sem Fins Lucrativos

Dessa forma, ao serem indagados se estaria dispostos a pagar para manter a EEP, $63 \%$ dos moradores responderam que sim e outros $37 \%$ revelaram-se não estar dispostos a pagar um valor mensalmente. Resultados similares foram obtidos por Silva et al. (2004), onde, $68 \%$ estariam dispostos a pagar pela preservação do parque 'Chico Mendes' situado na cidade de Rio Branco, no Acre.

Como resultado final de todo processo de estimação da DAP positiva total chegou-se a um valor médio de R\$21,47 por mês. Em termos comparativos, Mattos et al. (2006), estimou em $R \$ 27,98$ o valor mensal máximo para a população urbana de Viçosa, e Cirino et al. (2008), que estimaram em $R \$ 22,88$ mensais para a população da mesorregião dos Campos das Vertentes, no sudoeste do estado de Minas Gerais. Atualizando os valores encontrados, pelo Índice Geral de Preços da Fundação Getúlio Vargas, por Mattos et al. (2006) e Cirino et al. (2008), tem-se respectivamente $\mathrm{R} \$ 55,25$ e $\mathrm{R} \$$ 40,38 em valores de 2016.

A DAP mensal dos moradores foi encontrada multiplicando o valor de $\mathrm{R} \$ 21,47$ por $18.000,00$, que corresponde ao número estimado da população compreendida pelo raio de $30 \mathrm{~km}$ a partir da EEP, tem-se o valor econômico dos serviços ambientais providos pela EEP, sendo $R \$ 386.460,00$ por mês, ou ainda, $\mathrm{R} \$ 4.637 .520,00$ por ano.

Tabela 2: Coeficientes gerados pelo modelo logit para as variáveis que influenciam a disposição a pagar pela estação ecológica do Panga e seus efeitos marginais.

\begin{tabular}{|c|c|c|c|c|}
\hline VARIÁVEL & COEFICIENTE & Z & P $>$ I Z I & dy/dx \\
\hline Sexo & $(-) 0.1199914$ & $(-) 0.43$ & 0.670 & $(-0) .0265583$ \\
\hline Idade & $(-) 0.0274059$ & $(-) 2.10$ & $0.035^{* *}$ & $(-) 0.0060451$ \\
\hline dist_eep_km & $(-) 0.0255791$ & $(-) 1.06$ & 0.289 & $(-) 0.0056422$ \\
\hline temp_resid & 0.0517782 & 2.32 & $0.021^{* *}$ & 0.0114211 \\
\hline infra_urbana & 0.6024018 & 1.22 & 0.224 & 0.1417991 \\
\hline num_moradores & $(-) 0.0885297$ & $(-) 0.66$ & 0.511 & $(-) 0.0195277$ \\
\hline Irendafam & 0.7834809 & 2.12 & $0.034^{* *}$ & 0.0015173 \\
\hline Escola & $(-) 0.0226508$ & $(-) 0.18$ & 0.855 & $(-) 0.0049963$ \\
\hline Sustentabilidade & 20.145 .713 & 2.21 & $0.027^{* *}$ & 0.4606575 \\
\hline serv_ambi & 0.1298998 & 0.35 & 0.723 & 0.0282579 \\
\hline imp_preserv & 0.0832459 & 0.21 & 0.835 & 0.0185513 \\
\hline Eep & 0.5118422 & 1.21 & 0.227 & 0.1155906 \\
\hline eep_preservação & $(-) 0.205765$ & $(-) 0.50$ & 0.615 & $(-) 0.0459639$ \\
\hline eep_area_preserv & $(-) 0.7650512$ & $(-) 1.76$ & $0.078^{*}$ & $(-) 0.1693176$ \\
\hline out_atv_eep & $(-) 0.3212458$ & $(-) 1.09$ & 0.278 & $(-) 0.0700915$ \\
\hline
\end{tabular}




exp_eep_emp $\quad(-) 0.8953158$

$(-) 2.89+0.004 * * *$

$(-) 0.2046171$

Legenda: Significativa a ***1\%, significativa a **5\%, significativa a *10\%; *Efeito marginal da variável Irendafam foi calculado pelo comando margins do STATA.

Questionou-se aos entrevistados que não apresentaram DAP positiva, se um provável aumento de renda faria com que eles tivessem disposição a pagar. Assim, incluindo os questionários que apresentaram uma disposição a pagar caso houvesse um aumento da renda, o valor médio mensal da DAP passaria de $\mathrm{R} \$ 21,47$ para $\mathrm{R} \$ 22,81$. O valor agregado à Estação Ecológica por parte da sociedade seria de $\mathrm{R} \$ 410.580$ mensais ou $\mathrm{R} \$ 4.926 .960$ anuais. Considerou-se importante identificar quais são as variáveis que influenciaram a DAP. Dessa forma, a tabela 2 mostra os coeficientes que foram gerados com a estimação do modelo logit bem como o efeito marginal das variáveis utilizadas no modelo.

A variável idade apresentou sinal negativo, indicando que quanto maior a idade do indivíduo, menor a probabilidade de o mesmo apresentar disposição a pagar. O sinal foi de acordo com o esperado, uma vez que tem-se o entendimento de que a discussão ambiental é recente e os indivíduos mais jovens estão passando por um processo de conscientização pelo qual os mais idosos não passaram.

O sinal negativo para a variável idade também foi encontrado em outros estudos, como em Silva et al. (2004), Mattos et al. (2007), Cirino et al. (2008), Vieira (2009), e Börger (2013). Já Lo et al. (2015), ao aplicar a valoração econômica em árvores de grande representatividade estética no centro de Hong Kong, constataram que a variável idade apresentou coeficiente positivo.

Com a variável temp_resid (tempo de residência), tem-se que quanto maior o tempo em que indivíduo residia na localidade da entrevista, maior a sua probabilidade de o mesmo apresentar DAP positiva. A variável apresentou coeficiente positivo, de acordo com o sinal esperado, pois espera-se que os indivíduos que estão a mais tempo residindo nos locais de entrevista, apresentam maior DAP por conta do enraizamento que já estabelece com o local. Resultado semelhante foi encontrado em Obara (1999), onde concluiu-se que a variável tempo de residência era positivamente significativa para a DAP positiva. Vieira (2009) utilizou-se da questão em seu questionário, mas não utilizou da variável em seu modelo de regressão.

A variável Irendafam (renda familiar), apresentou coeficiente positivo, indo em conformidade com o sinal esperado, pois espera-se que quanto maior a renda maior a probabilidade de um indivíduo apresentar DAP positiva. Este resultado é similar aos encontrados nos trabalhos de Silva et al. (2004), Mattos et al. (2007), Cirino et al. (2008) e Börger (2013).

A variável sustentabilidade (conhecimento do que é sustentabilidade), apresentou coeficiente positivo, também em conformidade com o esperado para este sinal, uma vez que se imagina que o fato de o indivíduo apresentar maior conhecimento da problemática ambiental, existe uma probabilidade de o indivíduo apresentar DAP positiva. Silva et al. (2004) teve resultado semelhante ao estimar o coeficiente da percepção ambiental dos indivíduos, sendo este de coeficiente também positivo. Neste sentido, tanto o presente trabalho como o de Silva et al. (2004), buscou, com esta variável, estimar a percepção ambiental dos entrevistados e identificar se os mesmos estavam cientes da necessidade de preservação.

A variável eep_preservação (conhecimento de que a EEP é uma área de preservação) apresentou coeficiente negativo, sendo este diferente do sinal esperado, que era positivo. Esperava-se que ao saber que 
se tinha conhecimento de que era uma área de preservação, os entrevistados teriam maior probabilidade de apresentar uma DAP positiva. Fato que não se mostrou real, ou seja, existe uma diminuição na disposição a pagar pela preservação quando se tem o conhecimento de que a EEP trata-se de uma área de preservação ambiental.

A variável exp_eep_emp (indivíduos aceitariam que a estação fosse explorada), apresentou coeficiente negativo, em conformidade com o sinal esperado, pois imagina-se que caso o entrevistado aceitasse a exploração, teriam uma menor probabilidade de apresentar DAP positiva. A mesma variável foi utilizada por Obara (1999), mas não foi aplicada aos modelos econométricos de seus estudos.

A análise do efeito marginal das variáveis, nos mostram que, para a variável idade (idade), o aumento de um ano na idade do entrevistado diminui em $0,6 \%$ a sua probabilidade de apresentar DAP positiva. Em Mattos et al. (2007), o feito marginal da idade também se apresentou negativo, indicando que a elevação em um ano de vida reduz a probabilidade de pagamento em aproximadamente 0,38\%. Em Cirino et al. (2008), o efeito marginal também foi negativo, com uma redução em 0,07\% a probabilidade de DAP positiva. Em Silveira et al. (2013), o efeito marginal também foi negativo, com a probabilidade de redução da DAP em $0,54 \%$ com o aumento de um ano de vida do entrevistado.

O efeito marginal temp_resid(tempo de residência) foi positivo, no qual, com o aumento em um ano o tempo de moradia do entrevistado na localidade da entrevista, aumenta em 1,1\% a probabilidade de o mesmo apresentar DAP positiva. Em relação à variável Irendafam (logaritmo natural da renda familiar), a análise que pode ser feita do efeito marginal é que para aumentos de $1 \%$ na renda familiar, a probabilidade da DAP ser positiva aumenta em 0,0015p.p., conforme já enfatizado, Cirino et al. (2008) e Silva et al. (2004) também encontraram efeito marginal positivo para a renda.

No que tange ao efeito marginal da variável sustentabilidade (sustentabilidade), encontrou-se que ao conhecer o conceito de sustentabilidade, tem-se um aumento de 46p.p. a probabilidade de apresentar DAP positiva. Tal porcentagem é muito superior àquela encontrada em Silva et al. (2004), em que estimou um efeito marginal, também positivo, porém com uma porcentagem de 5,15p.p..

O efeito marginal da variável eep_preservação(o conhecimento de que a EEP é uma área de preservação), apresentou-se negativo, indicando que caso saiba de tal fato, apresenta uma porcentagem de 16,93p.p de probabilidade de não contribuir para a DAP positiva. A variável exp_eep_emp(indivíduos aceitariam que a estação fosse explorada), também apresentou efeito marginal negativo, em que, caso queira que seja explorado, apresenta uma probabilidade de 20,46p.p de não apresentarem DAP positiva.

Outra etapa da pesquisa, questionava sobre a Disposição a Receber dos entrevistados, sob a formulação de uma situação também hipotética: se a Estação Ecológica do Panga, estivesse munida de um recurso muito escasso no planeta e uma empresa estivesse disposta a explorar tal recurso, e, em contrapartida, pagasse um valor em dinheiro para poder explorar, você estaria disposto a receber para que o bem ambiental fosse explorado?. 
Em resposta, dos 300 questionados aplicados, 20\% apresentavam disposição a receber pela degradação e, por conseguinte, $80 \%$ não estavam dispostos a receber pela degradação. Em estimativas da DAR positiva, ao tirar a média dos valores declarados, obteve-se o valor de $\mathrm{R} \$ 47,21$ mensais por habitante. Esse valor, multiplicado por 18.000 , retorna um valor de $\mathrm{R} \$ 849.780$ mensais e $\mathrm{R} \$ 10.197 .360$ anuais. Tais valores mostram que os indivíduos inseridos em situação hipotética em que ocorre a perda dos bens ambientais, agregam valores altos para a medida compensatória.

Uma análise das variáveis que influenciam a DAR, na tabela 3 nos mostra que os homens estão mais dispostos a receber para preservar do que as mulheres, tal constatação ocorre por que a variável é positiva e representativa a 5\%. O coeficiente apresentou sinal esperado, uma vez que se imaginou que os homens estariam mais dispostos a abrir mão do ativo ambiental.

Tabela 3: coeficientes gerados pelo modelo logit para as variáveis que influenciam a disposição a pagar pela estação ecológica do Panga e seus efeitos marginais.

\begin{tabular}{|c|c|c|c|c|}
\hline VARIÁVEL & COEFICIENTE & $\mathbf{Z}$ & $P>|Z|$ & $\mathrm{dy} / \mathrm{dx}$ \\
\hline sexo & 0.7844821 & 2.23 & $0,026 * *$ & 0.1029079 \\
\hline idade & 0.3478 & 0.22 & 0,824 & 0.0004322 \\
\hline dist_eep_km & $(-) 0.140306$ & $(-) 0.48$ & 0,628 & $(-) 0.0017436$ \\
\hline temp_resid & $(-) 0.114314$ & $(-) 0.43$ & 0,669 & $(-) 0.0014206$ \\
\hline infra_urbana & 0.3870973 & 0.58 & 0,562 & 0.0429077 \\
\hline num_moradores & $(-) 0.649076$ & $(-) 0.41$ & 0,683 & $(-) 0.0080663$ \\
\hline Irendafam & $(-) 0.1314348$ & $(-) 2.94$ & $0,003^{*}$ & $(-) 0.163339$ \\
\hline escola & 0.1556932 & 0.99 & 0,32 & 0.0193486 \\
\hline sustentabilidade & $(-) 0.0058113$ & $(-) 0.01$ & 0,995 & $(-) 0.0007236$ \\
\hline serv_ambi & 0.1716891 & 0.39 & 0,699 & 0.0221272 \\
\hline imp_preserv & $(-) 1.16367$ & $(-) 2.62$ & $0,009 *$ & $(-) 0.19129$ \\
\hline eep & $(-) 1.106134$ & $(-) 2.01$ & $0,044 * *$ & $(-) 0.1569746$ \\
\hline eep_preservação & 0.38666 & 0.72 & 0,472 & 0.0506853 \\
\hline eep_area_preserv & 0.1023066 & 0.18 & 0,854 & 0.0127571 \\
\hline out_atv_eep & 0.0157875 & 0.04 & 0,966 & 0.0019601 \\
\hline exp_eep_emp & 0.1662812 & 4.32 & 0* & 0.2532658 \\
\hline
\end{tabular}

Legenda: Significativa a ***1\%, significativa a **5\%, significativa a *10\%; *Efeito marginal da variável Irendafam foi calculado pelo comando margins do STATA.

A variável da Irendafam (renda familiar) apresentou coeficiente negativo, conforme o esperado, pois entende-se que os entrevistados de menor renda apresentariam DAR positiva e constatando que com o aumento da renda familiar tem-se a uma maior probabilidade de a DAR ser negativa. Outra variável imp_preserv (importância de se preservar) apresentou coeficiente também negativo, conforme esperado, indicando que quanto maior o grau de importância que as pessoas agregam a preservação de áreas naturais existe uma menor disponibilidade a receber.

A variável eep (conhecimento da existência da EEP) aparece com coeficiente negativo, conforme esperado, indicando que quando se tem conhecimento da existência da EEP diminui-se a probabilidade de o indivíduo apresentar DAR positiva. A variável exp_eep_emp (estão de acordo com a exploração da EEP caso trouxesse emprego para a região),apresentou coeficiente positivo, sinal já esperado, uma vez que tem-se como pressuposto de que indivíduos que querem a exploração, são menos preocupados com as questões ambientais, logo gostariam de receber pela degradação. Conclui-se que os indivíduos que aceitariam que a estação fosse explorada apresentam uma maior disposição a receber.

Quanto à análise do efeito marginal da DAR, foi possível constatar, ainda pela tabela 3, que os homens estão mais propensos a receber em relação às mulheres; a variável Irendafam(logaritmo natural da 
renda familiar) que mostra que a influência da variação da renda familiar na DAR, indica que aumentos na renda familiar de $1 \%$ gera uma variação negativa na DAR de 0,0017p.p. Tal resultado está de acordo com o que se espera, ou seja, quanto maior a renda menor a DAR. Já o efeito marginal do conhecimento do entrevistado por parte da importância de se preservar (imp_preserv), mostra que se o mesmo reconhece essa importância, tem-se a probabilidade de 19,12p.p. do indivíduo não apresentar DAR positiva.

A variável eep (conhecimento do entrevistado sobre a existência da EEP), mostra que, quando ele conhece a EEP, as chances de ele ter DAR são negativas com probabilidade de 15,69\%. Entende-se que quanto mais a pessoa entende e conhece sobre o bem valorado, menores são as chances de a DAR ser positiva. A variável exp_eep_emp (estão de acordo com a exploração da EEP caso trouxesse emprego para a região), que indica a provável exploração da EEP para geração de empregos para a região, mostrou que as chances de ter uma DAR positiva elevam-se em 25

Diante do exposto, a valoração realizada no trabalho, apresentou-se importante por fornecer estimativas monetárias para a Estação Ecológica do Panga, podendo munir os gestores de informações relevantes quanto a sua estimativa monetária e dados socioeconômicos da população do entorno. Os valores encontradas mostram que o os objetivos do trabalho foram alcançados, Além de que a hipótese mostrou ser verdadeira, pois a sociedade, por meio da DAP positiva e DAR negativa, reconhecem a importância que a estação tem para a preservação e manutenção dos serviços ecossistêmicos.

\section{CONCLUSÃO}

As Estações Ecológicas (EE) são uma das unidades de conservação dentro do SNUC e apresenta-se como unidade de proteção integral. Em tais EE, só podem ser desenvolvidas atividades acadêmicas. É nesse contexto que se tem a EEP, situada no município de Uberlândia/MG, que tem como função a proteção de parte do Cerrado brasileiro. Assim, propôs-se um exercício valorativo que se mostrou importante por possibilitar, através da aplicação MVC, a compreensão de como a sociedade enxerga os benefícios ecossistêmicos da EEPanga.

Para tanto, determinou-se como amostra os moradores que são usuários indiretos dos benefícios ambientais decorrentes da EEP. Como características sócio econômicas, constatou-se que $59 \%$ eram do sexo feminino, $65 \%$ estavam em situação de matrimónio, $28 \%$ com segundo grau incompleto e tendo uma renda média mensal individual de R\$724,96 ao mês, sendo inferior ao salário mínimo da época, que estava fixado a $R \$ 788,00$. O grupo de moradores apresentaram uma taxa de $63 \%$ de DAP positiva, estimando um valor de $\mathrm{R} \$ 21,47$ por mês, elevado a população total, tem-se o valor de $\mathrm{R} \$ 386.460$ mensais, ou ainda, $\mathrm{R} \$ 4.637 .520$ por ano. Dentre as DAR positivas (20\%), foi possível estimar que $20 \%$ apresentavam disposição a receber pela degradação, com valores mensais individuais em $R \$ 47,21, R \$ 849.780$ mensais e $R \$ 10.197 .360$ anuais para a população total.

Ao estimar o modelo logit para identificar as variáveis que influenciam a DAP, verificou-se que idade (idade),temp_resid (tempo de residência), Irendafam (renda familiar) e sustentabilidade (sustentabilidade) 
foram significativas e, com exceção da variável idade, as demais tiveram sinal de acordo com o esperado. Dessa forma, pessoas mais velhas apresentaram menos DAP do que as mais jovens, quando o esperado era que tais pessoas apresentassem maior DAP. Verificou-se, que quanto mais tempo a pessoa mora no local, mais chances tinha de apresentar DAP positiva; Ter conhecimento sobre sustentabilidade elevavam as chances de a pessoa ter DAP, assim como uma maior renda familiar.

Para a DAR, ao estimar o modelo logit, notou-se que foram significativas as variáveis: $\operatorname{sexo}(\operatorname{sexo})$, eep (conhecimento da EEP), Irendafam (renda familiar) e exp_eep_emp (exploração da estação ecológica). Todas as variáveis apresentaram sinal de acordo com o esperado. Assim, pelos resultados, infere-se que os homens estão mais dispostos a receber para a degradação do que as mulheres. Pessoas com renda maior, com conhecimento da EEP tem menos chances de apresentar DAR positiva e a possibilidade de a EEP vir a ser usada economicamente aumenta as chances de a pessoa ter DAR positiva.

Diante do exposto, o método de valoração contingente apresentou-se importante por fornecer estimativa monetária para a Estação Ecológica do Panga, podendo munir os gestores de informações relevantes. As estimativas encontradas mostraram que existe sim uma preocupação da sociedade quanto a estação, e que a sua disposição a pagar só não é maior devido à baixa condição econômica em que muitos dos entrevistados estão submetidos. O valor estimado pode ter como consequência a sua utilização para a melhor gestão da estação, uma vez que os gestores vão saber as estimativas dos serviços ecossistêmicos agregados pela sociedade.

Para uma maior compreensão acerca da valoração econômica ambiental da EEP, tem-se como sugestão o desenvolvimento de valoração dos serviços ambientais com enfoque amostral no grupo de professores pesquisadores da Universidade Federal de Uberlândia, que utilizam da área para o desenvolvimento de suas atividades de pesquisas. Sua relevância estaria no fato de poder apresentar a valoração a uma estação ecológica, levando em consideração o público que desenvolvem pesquisa, reconhecendo a sua função enquanto área de produção científica. Este exercício valorativo, tornaria possível um debate maior acerca das diferentes percepções ambientais entre o grupo de moradores e o pesquisadores.

\section{REFERÊNCIAS}

ALVES, W. F.; ARAÚJO, A. A.; SILVA, C. R.. Valoração Ambiental: uma análise bibliométrica das produções acadêmicas no Brasil. In: CONGRESSO IBEROAMERICANO DE DESSAROLLO Y AMBIENTE, 7. Anais: Araraquara, 2015.

BARBISAN, A. O.; PANDOLFO, A.; REINEHR, R.; MARTINS, M. S; PANDOLFO, L. M.; GUIMARÃES, J.; ROJAS, J. W. J.. Técnica de valoração econômica de ações de requalificação do meio ambiente: aplicação em área degradada. Engenharia Sanitária Ambiental, v.14, n.1, p.119-128, 2009.

BENSUSAN, N.. Conservação da biodiversidade: em áreas protegidas. Rio de Janeiro: FGV, 2014.

BORGER, F. G.. Valoração econômica do meio ambiente: aplicação da técnica avaliação contingente no caso da Bacia do Guarapiranga. Dissertação (Mestrado em Ciência Ambiental) - Universidade de São Paulo, São Paulo, 1995.

BÖRGER, T.. Keeping up appearances: motivations for socially desirable responding in contigent valuation interviews. Ecological Economics, v.87, p.155-165, 2013.

BORGES, R. C.; GOMES, D. G.. Reserva Ecológica do Panga (Uberlândia/MG) - Proposta de Zoneamento e Contribuições ao Plano de Manejo. In: SEMINÁRIO DE INICIAÇÃO CIENTÍFICA, 13. Anais. Uberlândia: UFU, 2009.

CIRINO, J. F.; LIMA, J. E.. Valoração contingente da Área de Proteção Ambiental (APA) São José-MG: um estudo de caso. Revista de Economia e Sociologia Rural, v.43, p.649-674, 2008. 
DOUROJEANNI, M. J.; PÁDUA, M. T. J.. Biodiversidade: a hora decisiva. 2 ed. Curitiba: EDUFPR, 2007.

JÚNIOR, E. C.; COUTINHO, B. H.; FREITAS, L. E.. Gestão da Biodiversidade e Áreas Protegidas. In: GUERRA, A. J. T.; COELHO, M. C. N.. Unidades de Conservação. Rio de Janeiro: Bertrand Brasil, 2012. p.25-62.

KLINKE, C. A.; MACHADO, R.. A conservação do Cerrado brasileiro. Megadiversidade, Belo Horizonte, v.1, n.1, p.147155, 2005

LIMA, S. C.; BERNARDINO, A. R.. Mapeamento dos solos da Bacia do Ribeirão Panga. Sociedade \& Natureza, Uberlândia, v.4, p.77-84, 1992.

LO, A. Y.; JIM, C. Y.. Protest response and willingness to pay for culturally significant uran trees: Implications for Contigent Valuation Method. Ecologial Economics, v.114, p.58-66, 2014.

MATTOS, A. D. M.; JACOVINE, L. A. G.; VALVERDE, S. R.; SOUZA, A. L.; SILVA, M. L.; LIMA, J. E.. Valoração ambiental de Áreas de preservação permanente da microbacia do ribeirão São Bartolomeu no Município de Viçosa, MG. Revista Árvore, v.31, p.347-353, 2007.

MATTOS, K. M. C.; MATTOS, A.. Valoração Econômica do Meio Ambiente: uma abordagem teórica e prática. São Carlos: RiMa Editora, 2004.

MCCORMICK, J.. Rumo ao Paraíso. Rio de Janeiro: Relume Dumará, 1992.

MEDEIROS, R.. A proteção da natureza: das estratégias internacionais e nacionais às demandas locais. Tese
(Doutorado em Geografia) - Universidade Federal de Rio de Janeiro, Rio de Janeiro, 2003.

MOTTA, R. S.. Manual para Valoração Econômica de Recursos Ambientais. Brasília: MMA, 1998.

OBARA, A. T.. Valoração econômica de unidades e conservação: o método de valoração contingente. Tese (Doutorado em Ecologia e Recursos Naturais) - Universidade Federal de São Carlos, São Carlos, 1999.

PÁDUA, J. A.. Ecologia e Política no Brasil. Rio de Janeiro: IUPERJ, 2003.

PINDYCK, R. S.; RUBINFELD, D. L.. Econometria Modelos \& Previsões. Rio de Janeiro: Elsevier, 2004.

SILVA, R. G.; LIMA, J. E.. Valoração Contingente do Parque 'Chico Mendes': uma aplicação probabilística do método Referendum em Bidding Games. Revista de Economia e Sociologia Rural, Brasília, v.42, n.4, 2004.

SILVEIRA, V. C.; CIRINO, J. F.; PRADO FILHO, J. F.. Valoração econômica da área de proteção ambiental estadual da Cachoeira das Andorinhas - MG. Revista Árvore, v.37, n.2, p.257-266, 2013.

VIEIRA, P. F. S. P.. Valoração econômica das unidades de conservação de Serra de São José, MG: abordagem da disposição ao trabalho voluntário. Dissertação (Mestrado em Economia Aplicada). Universidade Federal de Viçosa, 2009.

VOLANOVA, S. R. F.; CHICHORRO, J. F.; ARRUDA, C. A. S.. Disposição a pagar pelo uso de unidades de conservação urbanas: Parque da Cidade Mãe Bonifácia, CuiabáMT. Interações (Campo Grande), v.11, n.1, p.43-53, 2010. 